\title{
Banknoten als sprachenpolitisches Instrument
}

\author{
Simon Pröll (München)
}

\begin{abstract}
For modern states, multilingualism is not an exception, but the norm. Most states explicitly deal with the status of their languages through their (often rather sparse) legislation, while implicitly reflecting their policy through language use on official documents. Banknotes provide a link between the official policy and its common application, as they are both a document of the state as well as an object of daily use. Here, the state is responsible for bridging the gap between legislature, national identity and the (sometimes conflicting) selfconceptions of its citizens. Thus, banknotes ideally provide evidence on a state's factual (rather than nominal) language policy. In addition, the textual and pragmatic functions of banknotes are not prone to change over time, which qualifies them as excellent sources for diachronic questions.

This article exemplarily illustrates the language policies of Norway, the Faroe Islands, Belgium and Luxembourg, as seen from a sociohistorical perspective, with their respective note emissions during their newer language history (19th and 20th centuries). It closes with a proposal for a typology of different language policies.
\end{abstract}

\section{$1 \quad$ Heranführung}

\subsection{Sprachnation und Sprachenpolitik}

Nach offensichtlichen, augenscheinlichen physiognomischen Merkmalen (wie Geschlecht oder Hautfarbe) ist die Sprache, die jemand spricht, das eindeutigste permanente Kriterium für die Zugehörigkeit zu einer Art Gruppe (cf. etwa Nelde/Labrie/William 1992: 404). Allerdings ist diese Art Gruppe notorisch schlecht bzw. zyklisch definiert, schließlich ist die einzige klare Gemeinsamkeit aller Sprecher des Deutschen, dass sie Deutsch sprechen - nicht aber, dass sie zusammengenommen eineindeutig den deutschen Staat definieren. In praktisch keinem aktuellen europäischen Staat ist die nationalromantische Idee einer Sprachnation (cf. Ammon 2000: 514), also eine exakte Übereinstimmung zwischen Territorium, Staatszugehörigkeit der Einwohner und Sprachgrenzen, umgesetzt (cf. Schreiner 2006: 43; Thomason 2001: 36-42). ${ }^{1}$ In den meisten Staaten war bzw. ist eine Form der Mehrsprachig-

\footnotetext{
${ }^{1}$ Die sogenannte Sprachnation war und ist dabei lediglich eine hilfreiche Vorstellung, um über ein klares Gruppenmerkmal eine gemeinsame Identität zu konstruieren, die es abgesehen von der gemeinsamen Sprache so gar nicht gäbe (vgl. auch Wright 2012: 62f.). Diese Ideologisierung von Sprache, vom Kommunikationsmittel hin zum Kulturträger, gipfelt schlussendlich in der (sprach-)nationalistischen Übersteigerung, dass "sich das 
keit gegeben; das ist nicht nur auf Migration zurückzuführen, sondern auch auf lange ansässige autochthone Sprachgruppen.

Latente innen- und außenpolitische Spannungen entzünden sich häufig an Fragen des nationalen Sprachgebrauchs. Zu einem Konflikt zwischen Sprachgruppen kommt es nach Nelde/Labrie/William (1992: 388) dann, wenn die Gruppen denselben Raum, "be it geographic, political, social, economic, cultural or religious in nature", besetzen und die Sprachen zum Symbol dieser Konkurrenz werden. Für Individuum, (Sprach-)Gruppe und Staat erwachsen daraus unterschiedliche, zum Teil gegenläufige Impulse. Im Folgenden wird näher betrachtet, wie staatliche Gremien über einen bestimmten Typus an Dokumenten, die sie herausgeben - ihre Banknoten -, sprachenpolitisch Stellung beziehen bzw. sprachplanerisch in Aktion treten.

\subsection{Banknoten als Schnittstelle und Quellenmaterial}

Die meisten modernen Staaten definieren den Status ihrer Sprachen explizit über ihre Legislative; jedoch ist die Umsetzung dieses Status in Form von konkreter Sprachplanung oftmals unterspezifiziert und problembehaftet: "Language policies, if they exist in some explicit form at all, tend to be chaotic, incongruent and extremely piecemeal" (Backhaus 2012: 227). Backhaus (2012: 226) führt nicht nur aus, dass die niedrigeren administrativen Level (diejenigen in vornehmlich direktem Bürgerkontakt) mit grundlegend anderen Problemen konfrontiert sind als die höheren staatlichen Institutionen, die den allgemeinen Status vorgeben, sondern auch, dass bezüglich der genauen Umsetzung "vor Ort" ein bedauerliches Forschungsdefizit besteht.

Banknoten bieten sich insofern als Quellenmaterial zur (teilweisen) Schließung dieser Lücke an, da sie eine Schnittstelle zwischen offizieller Sprachpolitik und "low level"-Bürgerkontakt darstellen. Wie etwa auch öffentliche Beschilderung (siehe z. B. Backhaus 2012: 240) legen sie implizit Zeugnis über die Sprachenpolitik ihrer Herausgeberinstitutionen (die nicht zwingend vollkommen staatlich sind, mindestens aber in enger Kooperation mit dem Staat agieren) ab. Als offizielle Dokumente haben Geldscheine eine klare Repräsentationsfunktion, sie stellen ein Aushängeschild ihrer Herausgeberländer nach außen dar. Sie leisten aber auch eine Repräsentation nach innen, wenn es um den Stellenwert konkurrierender Sprachen innerhalb desselben Währungsraums geht: Der Staat ist hier in der Verantwortung, den Spagat zwischen Legislative und dem (oft sehr uneinheitlichen) Selbstverständnis der Bürger auf repräsentative Weise zu überbrücken. In der Regel kommt man als Bürger nur relativ selten bzw. in sehr spezifischen Situationen in schriftlicher Form mit dem Staatsapparat in Kontakt. Geldscheine sind jedoch ein Gegenstand des täglichen Gebrauchs und in jeder der mannigfaltigen Situationen und Szenarien (staatlich wie privat) geläufig und selbstverständlich, in denen Geld eine Rolle spielt. Banknoten sind somit nicht nur ein Instrument der Sprachenpolitik einer Nation, sondern ebenso ein Symbol, ein Identifikationspunkt ihrer Einwohner. Interessant ist also nicht nur, was auf Geldscheinen steht, sondern auch in welcher Sprache. Dass die pragmati-

Sprachvolk durch Besinnung auf die eigene Sprache gleichsam auf sich selbst zubewegt (das Zu-sich-selbstKommen des Volksgeistes, seine Selbstbefreiung in der Sprache)" (Gardt 2000: 266, Hervorhebungen im Original). Wohin Verkürzungen dieser Argumentation/Position führen können, sei hier nur angedeutet: Die erste der 12 Thesen wider den undeutschen Geist, der nationalsozialistischen Flugschrift, die die Bücherverbrennungen im Jahr 1933 vorbereiten sollte, beginnt mit dem Punkt "Sprache und Schrifttum wurzeln im Volke".

ISSN 1615-3014 
sche (Text-)Funktion von Banknoten über die Zeit hinweg stabil bleibt, macht sie darüber hinaus auch diachron zu idealen Zeugen für die Entwicklung der Politik (und der Konflikte) ihrer Herausgeberländer. Anhand der Entwicklung ihrer Repräsentationsfunktion lässt sich nachverfolgen, welchen Stellenwert die verschiedenen Sprachen innerhalb eines Staats bzw. eines Währungsraums genießen.

\section{$2 \quad$ Einzelanalysen}

Für gezielte Einzelanalysen greife ich unter Bezug auf Scheine des 19. und 20. Jahrhunderts auf vier Beispiele jüngerer soziohistorischer Sprachgeschichte aus dem skandinavischen Raum und den Benelux-Ländern zurück, die jeweils einen anderen Typ von Mehrsprachigkeit illustrieren.

1. In Norwegen hat der historische Einfluss des Dänischen die ungewöhnliche Situation geschaffen, dass zu einer Nationalsprache zwei konkurrierende, offizielle Schriftvarianten nebeneinander existieren (und konkurrieren), die untereinander verständlich sind und regional unterschiedlich verbreitet sind.

2. Auf den Färöern hat sich - gegen ebenfalls dänischen Einfluss - die lange nur im privaten Raum mündlich tradierte Landessprache auch im öffentlichen, amtlichen und generell schriftlichen Gebrauch emanzipiert. Die Inseln sind heute offiziell einsprachig, in der Praxis herrscht aber Zweisprachigkeit vor.

3. Belgien ist offiziell dreisprachig, dabei aber auf Territorien aufgeteilt, die jeweils mehr oder weniger stark auf Einsprachigkeit angelegt sind.

4. Auch Luxemburg ist offiziell dreisprachig, dabei aber - ohne dass eine regionale Verteilung der einzelnen Sprachen vorläge - auch im Alltag konfliktfrei mehrsprachig. Die Landessprache hat sich in relativ kurzer Zeit zum Nationalsymbol ausgebaut.

\subsection{Norwegen}

Die heutige sprachliche Situation Norwegens erklärt sich zu großen Teilen aus seiner Historie: Ab 1349 ist Norwegen in Folge mehrerer großer Pestepidemien, der schätzungsweise bis zu zwei Drittel der Bevölkerung (und in hohem Maße auch die Herrscherklasse) zum Opfer fallen, wirtschaftlich in desolatem Zustand und zudem in weiten Landstrichen entvölkert. Norwegen ist damit zunächst in eine Union mit dem stärkeren Nachbarn Dänemark gezwungen, später verliert es vollständig seine Eigenständigkeit. Die meisten politischen - und nach der Reformation auch kirchlichen - Ämter werden mit Dänen bzw. mit Norwegern, die in Dänemark ausgebildet wurden, besetzt. Erst 1814 löst sich diese Verbindung und das mittlerweile wieder erstarkte Norwegen fällt Schweden zu; als Resultat des weiteren wirtschaftlichen Aufschwungs sowie der europaweiten nationalromantischen Bewegungen kommt es 1905 zur Unabhängigkeit Norwegens.

Die lange Zeit unter dänischer Krone, mit Dänisch in der Verwaltung und Dänisch in der Kirche hat weitreichende Folgen auf den norwegischen Sprachgebrauch: In den Ballungsräumen werden die norwegischen Mundarten stark "dänisiert". Im Rahmen der Unabhängigkeitsbestrebungen sowie als Begleiterscheinung der europaweiten nationalromantischen Bestrebungen im 19. Jahrhundert kommt es zum Bedürfnis, sich auch sprachlich von Däne- 
mark zu emanzipieren (cf. Vikør 2006: 1751). Hierfür gibt es zwei konkurrierende Ansätze: Zum einen wird versucht, die bestehende dänische Schriftsprache zu "norwegisieren", d.h. an die typische Aussprache der Norweger anzupassen. Diese Koiné wird zunächst Riksmål ("Reichssprache") genannt, ab 1929 Bokmål ("Buchsprache"). ${ }^{2}$ Zum anderen wird aber auch versucht, die archaischeren (west-)norwegischen Dialekte in ihren Gemeinsamkeiten zu erfassen. Eine zentrale Funktion nimmt hier der Sprachwissenschaftler und -planer Ivar Aasen (1813-1896) ein, der auf der Basis umfangreicher Datenerhebungen den Landsmål-Standard ("Landessprache") schafft, ab 1929 Nynorsk ("Neunorwegisch") genannt. Das moderne Norwegische kennt damit zwei offizielle Standards, jedoch nur für die Schriftsprache: Generell werden in Norwegen heute situationsunabhängig praktisch nur Dialekte gesprochen. ${ }^{3}$

Allerdings nähern sich die beiden Varianten über die Jahrzehnte hinweg - teils aus natürlicher Konvergenz, teils aus gezielter Sprachplanung mit mehreren Reformen (cf. dazu Vikør 2007: 196-235) - deutlich an, so dass sie sich heute, aus rein synchroner Perspektive, nur noch relativ wenig unterscheiden. Schon unter den verschiedenen festlandskandinavischen nordgermanischen Sprachen (Dänisch, Schwedisch, Norwegisch) ist das gegenseitige Verständnis äußerst hoch; die beiden norwegischen Schriftstandards sind im Prinzip zwei Optionen, die dialektale Variation Norwegens schriftlich zu erfassen und untereinander praktisch ohne Verständigungsprobleme austauschbar. Teil der Abschlussprüfung an norwegischen Schulen ist es, zu zeigen, dass man beide Standards auch aktiv schreiben kann. Hier sind, in den Worten Kurt Braunmüllers (1999: 345), "die Abstände zwischen den beiden Landessprachen gering, die historischen und ideologischen Barrieren dagegen hoch".

Das Problem der beiden Schriftsprachen Norwegens dreht sich also nicht um ansonsten blockiertes gegenseitiges Verstehen, sondern um verschiedene Ideologien (cf. Vikør 2007: 196), "zwei verschiedene Einstellungen, um ein unterschiedliches Selbstverständnis dessen, was 'norwegisch' ist" (Braunmüller 1999: 340; cf. detailliert auch Jahr 2007).

Im hier betrachteten sprachenpolitischen Kleinsystem findet diese Spaltung lange keinen Reflex: Bis 1983 (Scheinserien I-V) sind alle norwegischen Scheine komplett in Bokmål gehalten (cf. Madsen/Christensen 1983). Erst $1984^{4}$ ändert sich dies. Der Ursprung dafür ist wohl im politischen Klima der 1970er Jahre zu suchen, das in den 1980er Jahren zu einer aktiven Anti-Diskriminierungspolitik führte (cf. Nesse 2013: 186). Der neue 50-KronenSchein (der Schein mit dem kleinsten Nennwert) in Serie VI zeigt das Konterfei von Aasmund Olavsson Vinje, einem der ersten frühen Nynorsk- bzw. Landsmål-Vertreter. Auf diesem Schein taucht erstmalig die Nynorsk-typische Landesbezeichnung Noreg statt Norge (Bokmål) auf (Abbildung 1).

\footnotetext{
2 "Bokmål is derived from written Danish as pronounced by Norwegians" (Vikør 2006: 1751).

${ }^{3} \mathrm{Ob}$ es so etwas wie einen überregionalen, gesprochenen Standard gibt, wird kontrovers diskutiert (cf. z.B. die Beiträge in Jahr/Mæhlum 2009 oder Papayian 2012).

${ }^{4}$ Der norwegische Sprachenstreit läuft zu diesem Zeitpunkt schon über ein Jahrhundert, beide Varietäten sind bereits 99 Jahre lang zumindest offiziell rechtlich gleichgestellt. Seine höchste Akzeptanz hatte Nynorsk bereits zum Ende des zweiten Weltkriegs überschritten (mit ca. einem Drittel der Norweger, cf. Nesse 2013: 172), seitdem nehmen die Zahlen relativ kontinuierlich ab.
} 

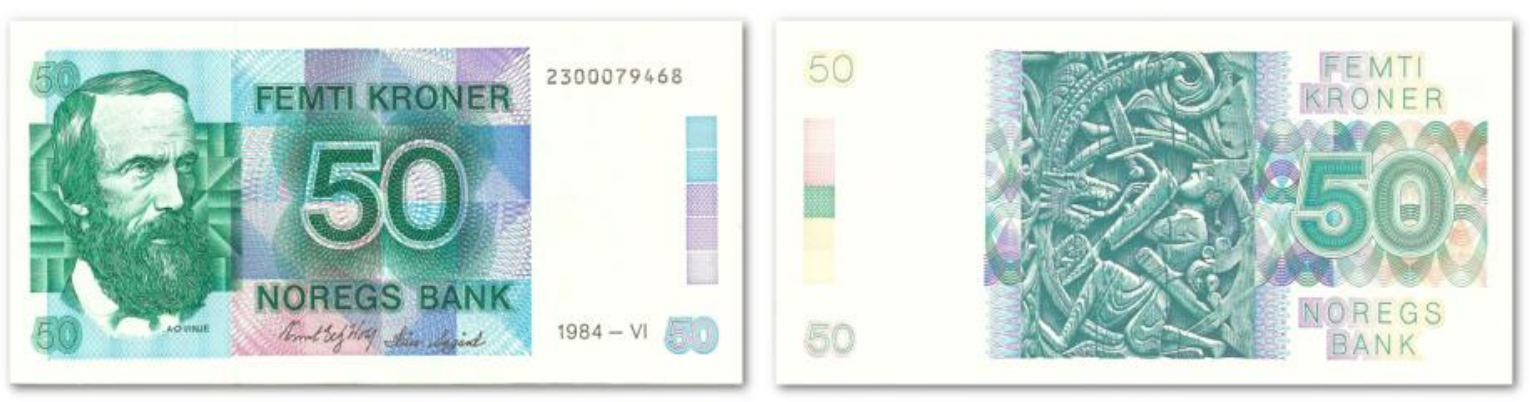

Abbildung 1: Norwegischer 50-Kronen-Schein von 1984, erstmals mit Landesbezeichnung Noreg

Für alle anderen Lexeme auf diesem Schein (und allen nachfolgenden) unterscheiden sich Bokmål- und Nynorsk-Formen nicht, allein die Schreibung des Landesnamens lässt einen Schluss auf die Schreibvarietät zu. Die darauf folgende Serie VII, ab 1994 herausgegeben und bis heute gültig, geht (unabhängig von der portraitierten Person) auf jedem Schein zu einem zweisprachigen Muster über, das bis heute gilt: Auf der Vorderseite steht Norges Bank (Bokmål), auf der Rückseite Noregs Bank (Nynorsk) (siehe Abbildung 2).
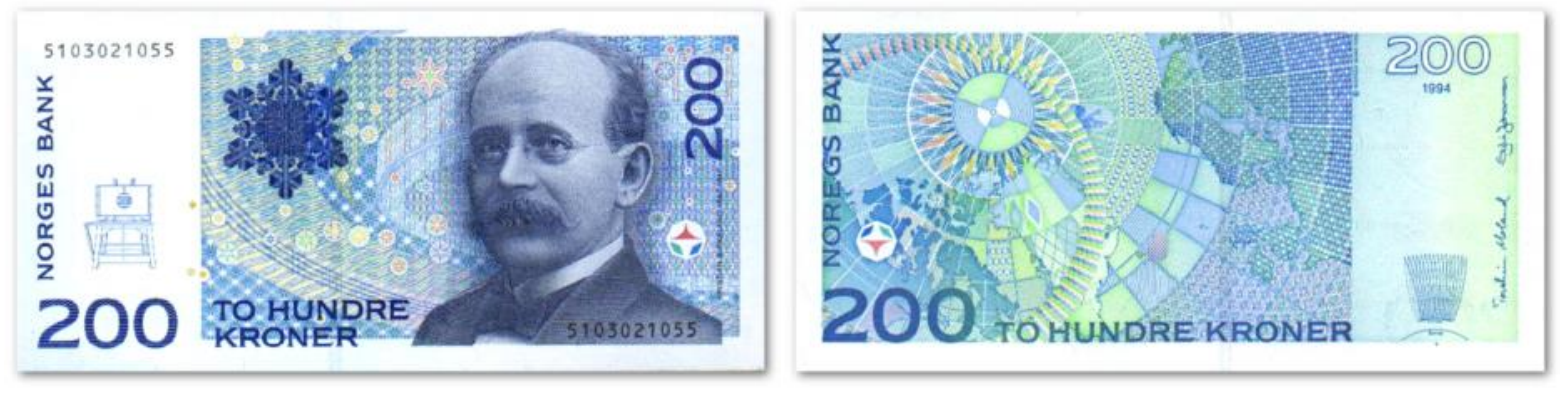

Abbildung 2: Norwegischer 200-Kronen-Schein, 1994; Vorderseite Bokmål (links), Rückseite Nynorsk (rechts)

\subsection{Färöer}

Die Färöer, eine Gruppe nordatlantischer Inseln, werden in der Hauptsache ab dem 9. Jahrhundert von Westnorwegen aus besiedelt (cf. Hagström 1984: 171; Poulsen 1997: 179). Die altwestnordischen Dialekte, die die Siedler mit sich bringen, wandeln sich über die Jahrhunderte hin zum modernen Färöischen. ${ }^{5}$ Bis in die Mitte des 19. Jahrhunderts hinein ist das Färöische (mit sehr wenigen mittelalterlichen Ausnahmen) nur mündlich tradiert. Erst dann wird eine eigene Orthographie dafür entwickelt, die sich nach und nach durchsetzt; dabei wird nach etymologischen Gesichtspunkten vorgegangen, ${ }^{6}$ das dialektal stark variable moderne Färöische steht daher schriftsprachlich dem Altwestnordischen bzw. dem vergleichbar entstandenen, in Bezug auf Wandelprozesse konservativen Isländischen sehr nahe (cf. Braunmüller 1999: 265, 267).

\footnotetext{
${ }^{5}$ Damit soll natürlich nicht ausgesagt werden, dass das moderne Färöische einheitlich wäre; die dialektale Vielfalt ist reichhaltig (cf. Hagström 1984: 175; Thráinsson et al. 2004: 339-368).

${ }^{6}$ Diese Ausrichtung ist zum einen der damaligen Zeit geschuldet, dem (spät-)romantischen bzw. (früh-)philologischen Interesse an historisierender Rekonstruktion (cf. Hagström 1984: 175), kann zum anderen aber auch als eine bewusste sprachtypologische Abgrenzung gegenüber dem konkurrierenden Dänischen, in dem die typischen Charakteristika des Altnordischen (vermutlich durch intensiven Sprachkontakt) schon deutlich abgeschwächt waren, gesehen werden. Gleichzeitig stützt es die dialektale Vielfalt, weil die Schreibung alle Dialekte ähnlich exakt (bzw. ähnlich abstrakt) wiedergibt und keinen bevorzugt (cf. Vikør 2007: 38).
} 
Wie Norwegen (s. o.) sind auch die Färöer ab 1380 Teil des dänischen Königreichs, aller administrative bzw. offizielle Sprachgebrauch ist damit dänisch. Die Reformation bestärkt den Status des Dänischen weiter: Bis Anfang der 1930er Jahre ist die Sprache in den Kirchen (und der Bibel) das Dänische, auch der Schulunterricht findet lange ausschließlich auf Dänisch statt (cf. Hoff 2012: 56; West 1972: 166-170). Im Privaten wird aber durchgängig Färöisch gesprochen. Das Land ist damit über Jahrhunderte hinweg zweisprachig (cf. Hagström 1992; Braunmüller 1999: 267), individuelle Zweisprachigkeit (und zwar sowohl mündlich als auch schriftlich) ist heute praktisch in der ganzen Bevölkerung der Regelfall (cf. u.a. Hagström 1992: 39; Vikør 2006: 1750) - auch deshalb, weil Färöisch zwar heute allgemeine Schulsprache, Dänisch aber gleichzeitig Pflichtfach für alle ist (cf. Braunmüller 1999: $331)$.

Nachdem Dänemark im zweiten Weltkrieg vom Deutschen Reich besetzt wird, werden die bereits weitgehend vom Festland isolierten Färöer eilig aus strategischen Gründen von Großbritannien besetzt. Die daher notwendig gewordenen ersten eigenständigen Scheine der Färöer sind in Dänisch gehalten; später werden sie eigens für die Färöer in England gedruckt, zunächst sind es aber schlicht dänische Scheine, die - ebenfalls auf Dänisch - mit einem Vermerk überdruckt werden, der ihre Gültigkeit auf die Färöer einschränkt (cf. West 1972: 179-180; Cuhaj 2008: 477-478; hier in Abbildung 3).

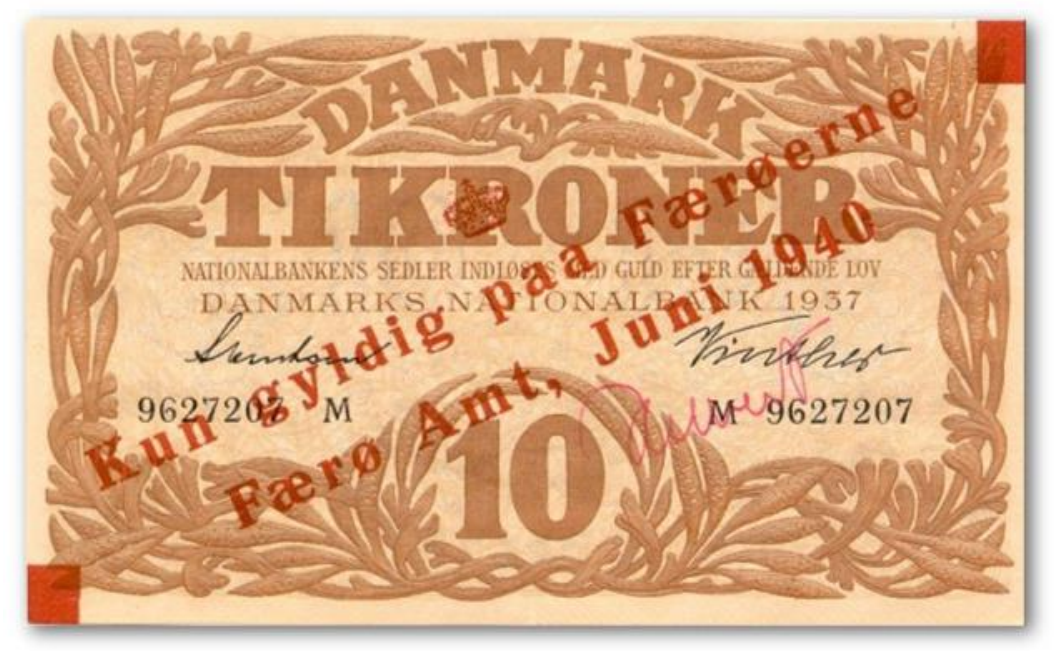

Abbildung 3: Dänischer 10-Kronen-Schein (Vorderseite) mit auf die Färöer begrenzter Gültigkeit, 1940 (Quelle: banknoteworld.com)

Die Eigenständigkeit während der britischen Besatzung bestärkt bestehende separatistische Tendenzen, die 1946 zu einer (positiven) Volksabstimmung über die Unabhängigkeit und in der Folge 1948 schließlich zum Heimastýrislógin, dem Gesetz über die Färöische Autonomie, führen.

Dies spiegelt sich in den Banknoten, die im Anschluss herausgegeben werden, entsprechend wider: Sie sind allesamt einsprachig färöisch, obwohl das Finanzwesen fortgesetzt auf das Engste mit Dänemark verquickt ist - bis heute gilt ein festgesetzter Umrechnungskurs von $1: 1$ zwischen dänischer und färöischer $\mathrm{Krone}^{7}{ }^{7}$ die färöischen Scheine werden von der

\footnotetext{
${ }^{7}$ Auch Grönland, ebenfalls autonomes Gebiet des Königreichs Dänemark und ökonomisch an die dänische Krone gebunden, wird 2006/7 die Erlaubnis erteilt, eigene Scheine in Umlauf zu bringen (Forslag til lov om
} 
dänischen Nationalbank gedruckt. Eigenes Münzgeld besitzen die Färöer nicht, es sind auch auf den Inseln ausschließlich dänische Münzen im Umlauf.

Dass die färöische Sprache für die Bevölkerung eine (laut Hagström 1984: 188 sogar die zentrale) Rolle für das kulturelle Selbstverständnis darstellt, zeigt sich auch darin, dass die Scheine mit dem höchsten Nennwert der jeweiligen Scheinserie einen direkten Bezug zur färöischen Sprache haben: Der 100-Kronen-Schein der ersten Serie, seit 1964 im Umlauf, zeigt Venceslaus Ulricus Hammershaimb (1819-1909), der die moderne Orthografie des Färöischen entwickelt hatte. Von 1980 bis 2005 ziert den 1000-Kronen-Schein der zweiten Serie das Konterfei von Jens Hendrik Oliver Djurhuus (1881-1948), der als erster moderner Autor der Inseln angesehen wird (so z.B. Isaksen 1993). ${ }^{8,9}$ Danach werden alle Scheine auf ein einheitlicheres Design mit Naturmotiven umgestellt.

Im Unterschied zur Situation in Norwegen geht es im färöischen Sprachenstreit tatsächlich auch ums Verstehen, aber in asymmetrischer Weise: Während praktisch alle Färinger zweisprachig sind, also problemlos Dänisch sprechen und verstehen, gilt dies nicht für die Dänen, die nur in Ausnahmefällen Färöisch beherrschen (cf. Hagström 1984: 181; dieses Muster einer Zweisprachigkeit ausschließlich beim schwächeren Part ist im Übrigen typisch für Sprachkontaktsituationen, cf. Thomason 2001: 66). Die Kenntnis des Färöischen ist damit ein Alleinstellungsmerkmal (aber auch ein Belastungsmoment) der färöischen Bevölkerung. Entsprechend müssen im Originaltext auf Färöisch verfasste Gesetze auch auf Dänisch übersetzt werden (da die Rechtshoheit immer noch beim Obersten Dänischen Gerichtshof liegt, cf. Vikør 2006: 1750); umgekehrt gilt das erst seit 1996 nicht mehr (cf. Poulsen 1997: 179).

\subsection{Belgien}

Belgien teilt sich heute in drei Regionen, das französisch- und deutschsprachige Wallonien im Süden, das niederländischsprachige Flandern im Norden, sowie die zweisprachige (französisch/niederländisch) Region der Hauptstadt Brüssel (cf. Die Verfassung Belgiens, Art. 3). Als Belgien sich 1830 unabhängig erklärt, wird (trotz offizieller Sprachfreiheit) überall zunächst Französisch alleinige Amtssprache, obwohl damals nur geschätzte 10 bis 15 Prozent der Bevölkerung - hauptsächlich der Oberschicht entstammend (cf. Mathiak 1993: 4f.) muttersprachlich französisch sind (cf. Halen 2003: 69). 1832 wird der Belgische Franc als Währung eingeführt. Zunächst sind alle Scheine ausschließlich Französisch beschriftet, ab 1886/7 wird die Rückseite der beiden kleinsten Scheine (20 und 50 Francs) Niederländisch (cf. Cuhaj 2008: 117). Während des 19. Jahrhunderts verläuft die ökonomische Entwicklung

\footnotetext{
pengesedler $i$ Grønland). Im Autonomiegesetz von 2009 (Lov om Grønlands Selvstyre) wird darüber hinaus Grönländisch als "offizielle Sprache" festgelegt ("§ 20. Det grønlandske sprog er det officielle sprog i Grønland."). Dennoch entscheidet sich die (zwischenzeitlich umgebildete) grönländische Regierung nach mehrjährigen Vorbereitungen der Motive - und einigen Verzögerungen - dagegen, eigene Scheine herauszugeben.

8 "Han ville bevise, at færøsk var et kultursprog blant andre kultursprog og ikke en »babarisk bjergdialekt «, som man havde hævdet fra dansk side, og et sprog, man kunne skrive hexametre i." ["Er wollte beweisen, dass Färöisch eine Kultursprache unter anderen Kultursprachen sei und kein »barbarischer Bergdialekt«, wie man es von dänischer Seite behauptet hatte, und eine Sprache, in der man Hexameter schreiben konnte", meine Übersetzung.] Isaksen (1993: 34)

${ }^{9}$ Über die Historie der färöischen Scheine informiert die Dänische Nationalbank direkt auf ihrer Homepage (http://www.nationalbanken.dk/DNDK/money.nsf/side/Faeroeernes_tidligere_seddelserier!OpenDocument).
}

ISSN 1615-3014 
des Nordens und des Südens unterschiedlich; Französisch zementiert sich als Sprache der oberen Schichten, des Wohlstands und damit auch des Bildungswesens. Dies führt unter anderem dazu, dass im Ersten Weltkrieg angeblich die einfachen Soldaten, die niederländisch sprechen, die Offiziere aus der französischsprachigen Oberschicht nicht verstehen (cf. Halen 2003: 72). Die Gerüchte - ob fundiert oder nicht - um daraus resultierende Tote verstärken den schon länger schwelenden Konflikt um die faktische Unterdrückung des Niederländischen. In der Folge des ersten Weltkrieges bzw. des Versailler Vertrages werden zusätzlich primär deutschsprachige Gebiete in Ostbelgien angeschlossen - es wird schlussendlich beschlossen, das Land in Sprachregionen zu gliedern. Um diesen Zeitpunkt herum breitet sich das Muster Vorderseite Französisch / Rückseite Niederländisch auch auf die größeren Nennwerte aus (cf. Cuhaj 2008: 118-126).

Nach dem Zweiten Weltkrieg kommt es zur Abstimmung über die Monarchie, die während der deutschen Besatzung eine umstrittene Rolle innehatte. Während die katholischmonarchistisch ausgerichteten Flamen mehrheitlich für ihre Beibehaltung stimmen, votieren die sozialistischeren Wallonen gegen die Monarchie (cf. Halen 2003: 75). Dies führt Belgien in der Folge weiter in die Krise; in den 1960er Jahren überholt darüber hinaus das vormals schwächere Flandern den wallonischen Süden in Bezug auf sein Wirtschaftswachstum. Hier wird deutlich, dass der Konflikt höchstens oberflächlich betrachtet ein Sprachkonflikt ist: Er dreht sich um Gleichberechtigung, um wirtschaftliche und gesellschaftliche Macht, um Religion. Sprache dient hier lediglich als scheinbarer gemeinsamer Nenner der Konfliktparteien. Sie ist aber gleichzeitig auch Abbild der Machtverhältnisse: Ab 1961 - zeitgleich mit der Veröffentlichung einer rechtsgültigen niederländischen Version der Verfassung, cf. Mathiak (1993: 33) - endet die strikte Fixierung des Französischen auf die Scheinvorder- und des Niederländischen auf die Scheinrückseite: Erstmals treten auch Scheine mit umgekehrter Konfiguration auf, in jeder neuen Serie folgt seitdem ein Teil der Nennwerte dem einen, der andere Teil dem anderen System (cf. Cuhaj 2012: 114-117, hier ein Beispiel in Abbildung 4).
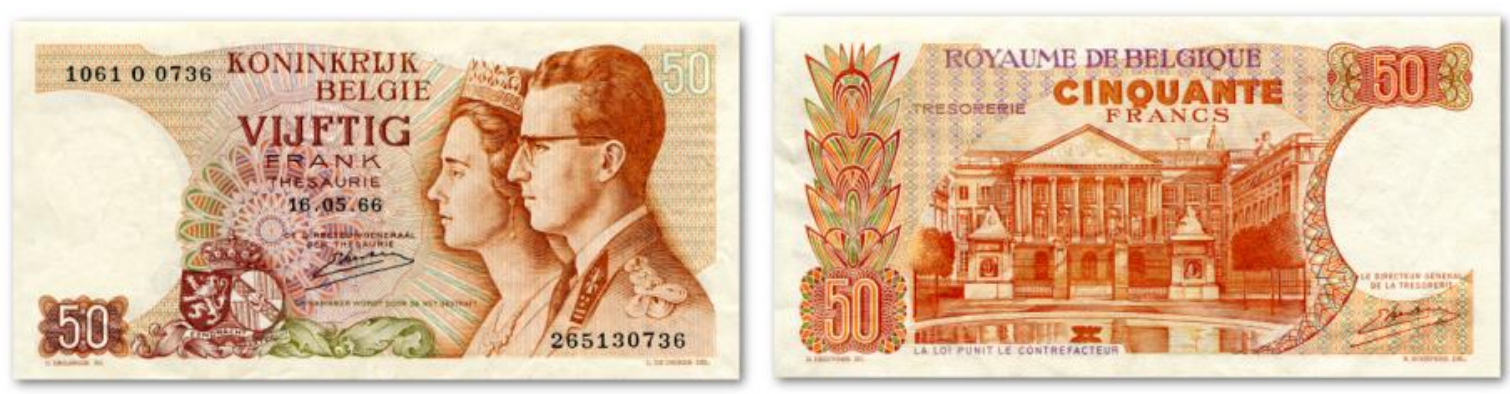

Abbildung 4: Belgischer 50-Franken-Schein von 1966; niederländische Vorderseite (links), französische Rückseite (rechts)

Das Territorialitätsprinzip, die Aufteilung des Landes in zunächst zwei (scheinbar) einsprachige Teilregionen bereits vor dem Zweiten Weltkrieg kann den Konflikt langfristig nicht entschärfen. Im Gegenteil bringen mehrere weitere Reformen eine Verfestigung der gegensätzlichen Positionen mit sich. Vor allem die Sprachgesetzgebung von 1962/63 ist folgenreich, da sie das Land faktisch in vier Areale teilt: Den niederländischen Norden, den französischen Süden, die zweisprachige Hauptstadt Brüssel sowie den (kleinen) deutschen Osten (siehe Abbildung 5). Seit den 1970er Jahren werden politische Parteien in einen französischsprachigen und einen niederländischsprachigen Regionalteil separiert; die fort- 
schreitende Föderalisierung bis in die 1990er Jahre hinein, die unter anderem zur Autonomie der Bildungssysteme und eigenen Parlamenten führt, geht einher mit der allmählichen, aber fortschreitenden bewussten Abschaffung dezidiert zweisprachiger Einrichtungen (cf. Halen 2003: 79). ${ }^{10} \cdot 11$ Das führt dazu, dass heute zur innerstaatlichen Kommunikation über die Territorialgrenzen zunehmend Englisch als lingua franca eingesetzt wird.

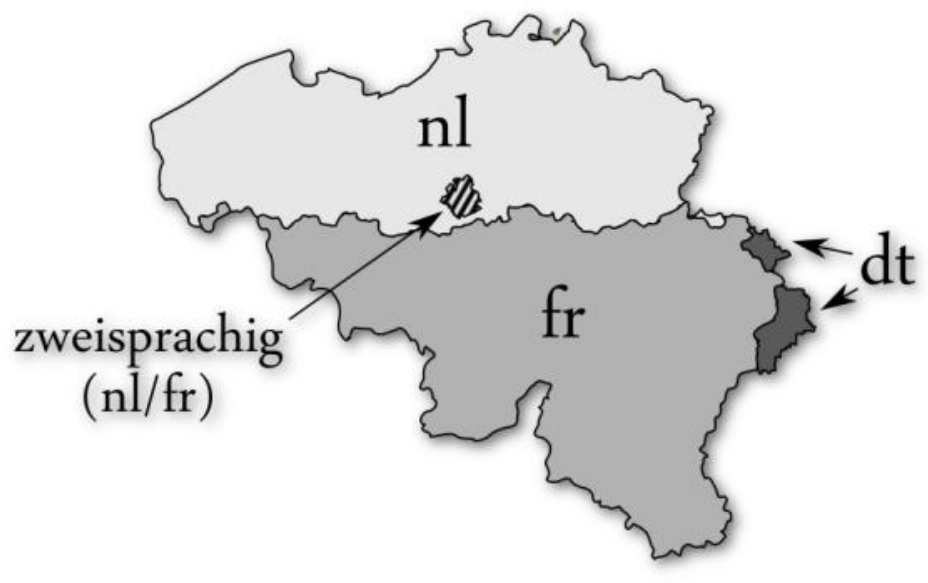

Abbildung 5: Sprachregionen Belgiens.

Im Jahr 1991 wird auch eine deutschsprachige Version der Verfassung gültig (cf. Witt 2001: 41) - ab 1992 tritt in der Folge auch das von den Sprecherzahlen her vergleichsweise marginale Deutsche ${ }^{12}$ auf Banknoten in Erscheinung: Die Vorderseite ist einsprachig, je nach Nennwert im Wechsel Französisch oder Niederländisch, die Rückseite teilen sich das Deutsche und die jeweils andere Sprache (vgl. Abbildung 6).
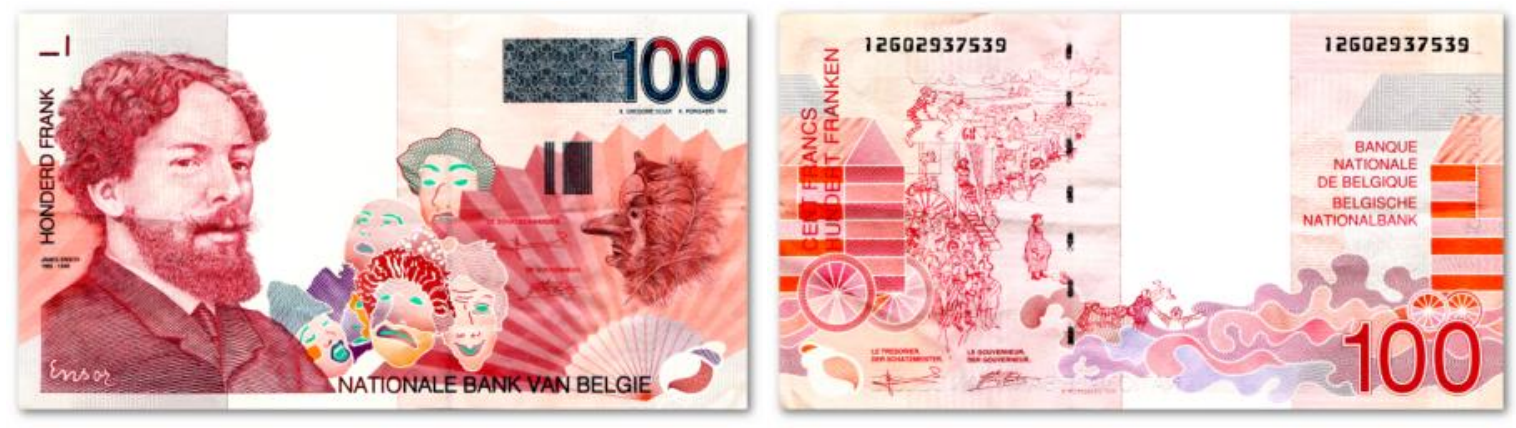

Abbildung 6: Belgischer 100-Franken-Schein nach 1992; niederländische Vorderseite (links), französisch/deutsche Rückseite (rechts)

Die belgische Politik ist damit offenbar streng bemüht, der Bevölkerung die Gleichberechtigung ihrer Sprachgruppen entsprechend der aktuellen Rechtslage zu signalisieren nicht aber, ihr vorwegzugreifen.

Mit der Einführung des Euro im Jahr 2002 (Belgien gehört zu den ersten 12 Euro-Beitrittsländer) ist der Streit zwischen Flamen und Wallonen nicht mehr auf Banknoten präsent; der

\footnotetext{
10 Das gilt im Übrigen auch für die belgischen Streitkräfte, die seit 2010 nur noch einsprachige Einheiten haben.

${ }^{11}$ Das deutsche Sprachgebiet ist jedoch keine eigene Region, es gehört administrativ zum französischen Teil; "Deutsch ist jeweils nur ko-offizielle regionale Amtssprache" WITT (2001: 39).

12 Willemyns (2006: 1759) zufolge entspricht die Deutsche Gemeinschaft 0,6 \% der Belgischen Bevölkerung.
} 
Konflikt selbst ist aber alles andere als beigelegt. Von 2007 bis 2012 führt der innerbelgische Konflikt zu wiederholten Regierungskrisen, eine baldige Lösung ist nicht in Sicht.

\subsection{Luxemburg}

Während in Norwegen und den Färöern die Alltagskommunikation in der Hauptsache einsprachig stattfindet, herrscht im Großherzogtum Luxemburg, ebenfalls seit dem 19. Jahrhundert unabhängig, Mehrsprachigkeit vor. ${ }^{13}$ Heute koexistieren drei offizielle Sprachen: Deutsch, Französisch und Luxemburgisch (Eigenbezeichnung Lëtzebuergesch). Das Luxemburgische entsteht sprachhistorisch gesehen als Teil des (hoch-)deutschen Dialektkontinuums, genauer gesagt des Moselfränkischen. Im Gegensatz zu den moselfränkischen Dialekten auf deutschem Staatsgebiet wird es im Laufe der Zeit mit einer eigenständigen Orthographie ausgestattet, statt - wie dies für bundesdeutsche Dialekte auch heute noch gilt - vom Schriftdeutschen orthographisch "überdacht" zu werden. Diese Standardisierung ist in der anhaltenden Diskussion zum Status derartiger sprachhistorischer Entwicklungen nach wie vor eines der Hauptargumente dafür, Luxemburgisch als eigenständige Sprache anzusehen (so z.B. Ammon 2000: 515; Janson 2012: 131f. z. B. schlägt dagegen einen metalinguistischen Zugang vor).

Die Scheine der luxemburgischen Privatbanken des 19. Jahrhunderts sind zunächst teils Deutsch, teils Französisch; die Banknoten des Staats des Großherzogtums Luxemburg ab 1914, während der deutschen Besatzungszeit im Ersten Weltkrieg, haben dann eine französischsprachige und eine deutsche Seite, die der Internationalen Bank in Luxemburg sind komplett deutsch (cf. Weiller 1981; Grabowski/Huschka/Schamberg 2006: 184-189). Bis 1919 ist Luxemburg Mitglied im Deutschen Zollverein, durch Artikel 40 des Versailler Vertrags wird dies aufgehoben. Es positioniert sich in der Folgezeit offiziell als neutral, sympathisiert jedoch mit dem französischen Nachbarn, auch aus (berechtigter) wachsender Sorge vor dem Deutschen Reich: Von den deutschen Nationalsozialisten wird Luxemburg als deutsches Gebiet erachtet (cf. Krier 1976: 628). Über die 20er und 30er Jahre bleibt es dennoch beim Muster einer französischen Vorder- und einer deutschen Rückseite der Scheine (Francs / Franken). Im Mai 1940 wird Luxemburg vom Deutschen Reich besetzt. Bezeichnenderweise werden direkt vor der Besatzung Scheine gedruckt (10 Frang, 20 Frang) bzw. geplant (5 Frang), die die Landesbezeichnung und den Nennwert auf beiden Seiten ausschließlich auf Luxemburgisch zeigen (LETZEBURG, ZENG FRANG), nur der Straftext ist auf Deutsch und auf Französisch; sie kommen nicht mehr in Umlauf und werden von den Besatzern vernichtet (cf. Weiller 1981: 130-131; Grabowski/Huschka/Schamberg 2006: 189).

Im August 1940 wird der Gebrauch der französischen Sprache verboten (cf. Kramer 1996: 130). Ein Versuch im Oktober 1941, mittels eines "Zensus" der Muttersprache, Staatszugehörigkeit und Volkszugehörigkeit eine Annexion zu legitimieren und die Luxemburger als offensichtlichen Teil des Deutschen Reichs darzustellen, scheitert: Entgegen der beabsichtigten Positionierung zugunsten des Deutschen (sowohl in sprachlicher als auch "völkischer" Hinsicht) zeichnet sich ab, dass ein überwältigender Teil der Bevölkerung dreimal "Luxemburgisch" angeben würde. Dies zwingt den Gauleiter dazu, die Aktion abzubrechen (cf. Berg

\footnotetext{
${ }^{13}$ Das ist nicht nur eine historisch gewachsene Situation (cf. Berg 1993: 9-18), sondern wird aktuell auch dadurch gefördert, dass mehr als die Hälfte der Arbeitnehmer im Land lebende Ausländer oder Arbeitsgrenzgänger sind (cf. Hansen-Pauly 2003: 85).
}

ISSN 1615-3014 
1993: 17-18; Hansen-Pauly 2003: 87), was im Umkehrschluss die sprachlich unterstützte luxemburgische Selbstabgrenzung psychologisch stärkt. ${ }^{14}$ Spätestens zu diesem Zeitpunkt wendet sich das sprachliche Selbstverständnis der Luxemburger endgültig davon ab, Sprecher eines deutschen Dialekts zu sein, die rasante Emanzipation des Lëtzebuergeschen zur eigenständigen Sprache ist somit auch ideologisch stark motiviert.

Dennoch wird Luxemburg im August 1942 annektiert. Während der Besatzungszeit ist deutsches Geld im Umlauf (cf. Weiller 1981: 31; Grabowski/Huschka/Schamberg 2006: 189). 1944 wird Luxemburg von der NS-Besatzung befreit, das Deutsche wird in der Folge im offiziellen Raum gemieden: Entsprechend ändert man den Namen des Landes im internationalen Raum von Luxemburg zu Luxembourg. Die meisten Scheine der Reihen ab 1944 sind vorne Französisch, hinten Luxemburgisch, manche komplett Französisch, kein einziger mehr Deutsch. Lediglich für einen Fofzeg Frang-Schein dieser Serie existiert eine Variante, die noch einen deutschen Straftext auf der Rückseite aufführt: Dieser wird aber mit einem Muster überdruckt und damit unleserlich gemacht (cf. Weiller 1981: 134).

Wie in Belgien ist der Gebrauch des Französischen über längere Zeit hinweg prestigeträchtig (cf. für einen knappen tabellarischen Überblick über die Geschichte der luxemburgischen Sprachpraxis Hansen-Pauly 2003: 87-93, außerdem Fehlen 2013), was begünstigt, dass mangels gesetzlicher Regelungen - für die es lange keinen Anlass gibt ${ }^{15}$ - "de facto das Französische die Amtssprache Luxemburgs" (Witt 2001: 48; cf. auch Fehlen 2013: 44-48) ist. Entsprechend sind die ab 1953 herausgegebenen Banknotenreihen vollständig Französisch gehalten (cf. Weiller 1981: 132-159). Erst ab 1980 kehrt man zur luxemburgischen Rückseite zurück, nun mit dem Landesnamen in der zwischenzeitlich neu standardisierten Orthografie (LËTZEBUERG, HONNERT FRANG, cf. Cuhaj 2012: 623-625, hier Abbildung 7) - das kann als Zeichen einer zunehmenden "Verwendung des Lëtzebuergeschen als Medium schriftsprachlicher Kommunikation" (Berg 1993: 156), also als langsame Übernahme auch des schriftlichen Geltungsbereichs der neuen Nationalsprache dienen. Am Sprachdesign der luxemburgischen Scheine ändert sich dann bis zur Einführung des Euro nichts mehr.
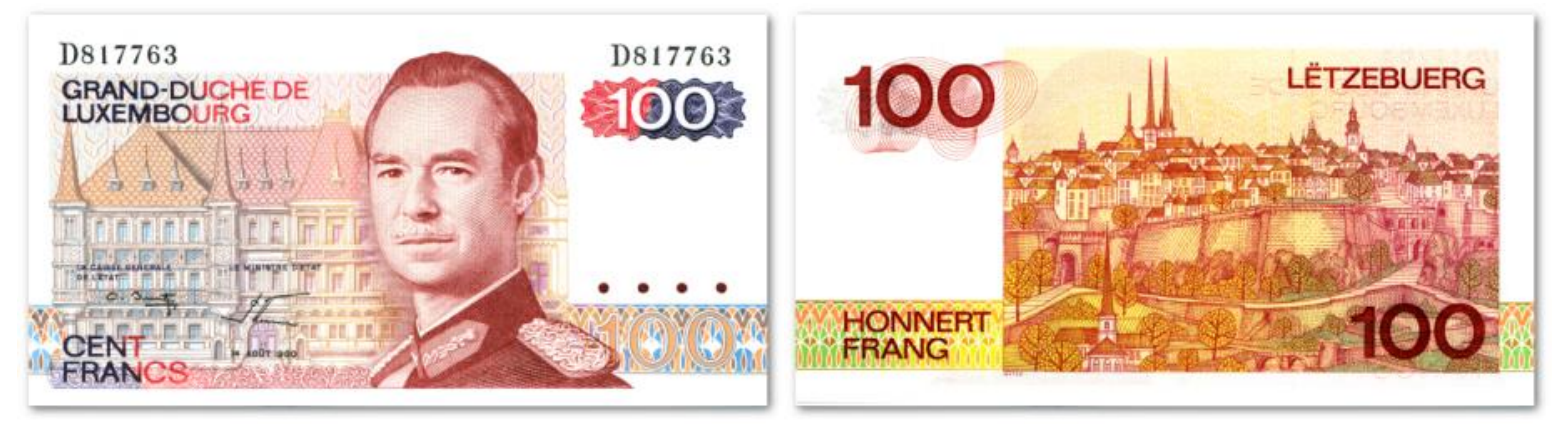

Abbildung 7: Luxemburgischer 100-Franken-Schein, 1980; französische Vorderseite (links), luxemburgische Rückseite (rechts).

\footnotetext{
${ }^{14}$ Dass auch die Exilregierung von London aus mittels BBC auf Luxemburgisch mit ihrer Bevölkerung kommuniziert, trägt weiter dazu bei, den Zusammenhang zwischen Sprache und Nation zu stärken (cf. Hansen-Pauly 2003: 87).

15 "Die Mehrsprachigkeit war eigentlich immer so selbstverständlich, dass man keine offiziellen Regelungen brauchte." (Hansen-Pauly 2003: 86)
} 
1984 wird schließlich ein Sprachengesetz (Loi du 24 février 1984 sur le régime des langues au Luxembourg) verabschiedet, das den rechtlichen Status der drei im Alltag meistgenutzten Sprachen regelt: Luxemburgisch ist seitdem offiziell National- und Amtssprache, Französisch die Sprache der Legislative und - ebenso wie das Deutsche - Amtssprache. Ursache für das Treffen einer offiziellen Regelung ist jedoch kein Sprachenstreit wie im benachbarten Belgien, sondern die Anforderungen, die der internationale Kontakt an den Staat Luxemburg stellt (cf. Hansen-Pauly 2003: 86, 91).

\section{$3 \quad$ Schluss}

Der Vergleich dieser vier ausgewählten ${ }^{16}$ Kontaktsituationen im Rahmen nationalstaatlicher Mehrsprachigkeit zeigt, dass sich der Status, den die jeweils genutzten Sprachen genießen, in seiner gezielten Verwendung in staatlich-offiziellen Alltagssituationen - wie eben auf Banknoten - klar widerspiegelt; zugleich aber auch, dass die Verwendung eben diesen Status stützt und erweitert, schließlich ist Schriftlichkeit bzw. volle Funktionalität ein Kriterium, "eigenständige" Sprachen von "unselbstständigen" Dialekten zu unterscheiden (cf. etwa Janson 2012: 132): So wurde etwa Färöisch zur Mitte des 19. Jahrhunderts von offizieller Seite als dänischer Dialekt betrachtet, mit der Begründung, dass keine schriftliche Fixierung existiere (cf. Hoff 2012: 53). Auch dass sich das Luxemburgische innerhalb relativ kurzer Zeit vom Image eines regionalen Dialekts zu dem einer voll ausgebauten Sprache entwickeln konnte, ist sicherlich sowohl der identitätsstiftenden Funktion wie auch der bewussten Expansion in offizielle Funktionskontexte zuzuschreiben. Status und offizieller Gebrauch stehen klar in einem positiven Rückkopplungsverhältnis.

Zur Beschreibung des staatlichen Umgangs mit interner Mehrsprachigkeit bietet sich, ausgehend von den vorliegenden Beispielen, eine vereinfachte Typologie mittels zwei (nicht ganz trennscharfen) Achsen an (vgl. Abbildung 8): Einer Achse, die den Grad der Aktualitätsreflexion erfasst (senkrecht), und einer, die die Konfliktbereitschaft charakterisiert (waagrecht).

\footnotetext{
${ }^{16}$ Ein weiterer Problemkomplex, der unterschiedlicher Alphabete, soll hier nur schlaglichtartig erwähnt werden; der Zerfall der sozialistischen Staaten in Osteuropa im späten 20. Jahrhundert führt hier oftmals zu komplexen sprachpolitischen Situationen. Das ehemalige Jugoslawien ist offiziell dreisprachig, wobei das Serbokroatische auch auf Banknoten - in zwei Varianten erfasst ist, einer in lateinischen und einer in kyrillischen Lettern. In den Nachfolgestaaten wird das Serbokroatische zur einzigen, lateinisch transliterierten Amtssprache Kroatiens bzw. zur einzigen, aber auf beide Arten transliterierten Amtssprache Serbiens.

Im heutigen Moldawien, bis 1989 Republik der UdSSR, ist Rumänisch alleinige Amtssprache. Es wird seit dem 19. Jahrhundert zunächst kyrillisch verschriftet, in der Folge der moldawischen Unabhängigkeit jedoch auf lateinische Schrift umgestellt, was sich auch in den Banknoten wiederspiegelt (cf. Cuhaj 2012: 686-688). Darüber hinaus wird die Sprache zwischen 1994 bis 2013 offiziell "Moldauisch" genannt, seitdem wieder "Rumänisch". Die völkerrechtlich nicht anerkannte Region Transnistrien, die sich 1992 von Moldawien abtrennt, bewahrt die kyrillische Transliteration des Rumänischen (auch auf Banknoten, die ohnehin zunächst modifizierte gebrauchte russische Rubel-Noten sind, cf. Cuhaj 2012: 1006-1009) - weiterhin zählen dort auch Russisch und Ukrainisch (beide ebenfalls kyrillisch geschrieben) zu den Amtssprachen (cf. Steinke 2006).
}

ISSN 1615-3014 


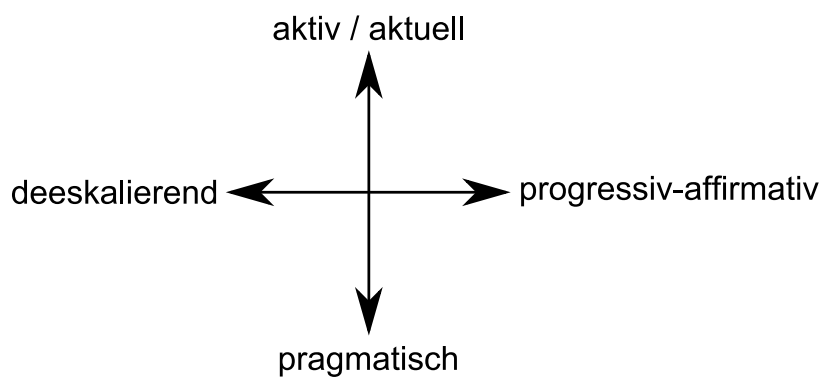

Abbildung 8: Typologieachsen zur internen Mehrsprachigkeit

Während die Färöer klar im Quadranten rechts oben verortet werden müssten, weil die Politik den gesellschaftlichen Trend progressiv-affirmativ mitgestaltet, und dabei eine aktuelle, aktive Rolle einnimmt, ist der Belgische Weg zwar ebenfalls auf Aktualität bedacht, aber auf eine definitiv deeskalierende Art. Norwegen verfolgt dagegen den pragmatischen Zugang, erst gesellschaftlich bereits weitgehend Etabliertes widerzuspiegeln. Für Luxemburg lässt sich so für die letzten Jahrzehnte gar eine Änderung der Politik, weg vom aktiv-gestalterischen zum pragmatisch-neutralen Bereich konstatieren.

\section{Literatur}

Ammon, Ulrich (2000): "Sprache - Nation und die Plurinationalität des Deutschen". In: Gardt, Andreas (ed.) (2000): Nation und Sprache. Die Diskussion ihres Verhältnisses in Geschichte und Gegenwart. Berlin/New York, de Gruyter: 509-524.

Backhaus, Peter (2012): "Language policy at the municipal level". In: Spolsky, Bernard (ed.) (2012): The Cambridge Handbook of Language Policy. Cambridge, Cambridge University Press: 226-242.

Berg, Guy (1993): "Mir wëlle bleiwe, wat mir sin." Soziolinguistische und sprachtypologische Betrachtungen zur luxemburgischen Mehrsprachigkeit. Tübingen: Niemeyer.

Braunmüller, Kurt ( $\left.{ }^{2} 1999\right)$ : Die skandinavischen Sprachen im Überblick. Tübingen/Basel: Francke.

Coulmas, Florian (1985): Sprache und Staat. Studien zu Sprachplanung und Sprachpolitik. Berlin/New York: de Gruyter.

Cuhaj, George S. ( $\left.{ }^{12} 2008\right)$ : Standard Catalog of World Paper Money. General Issues. 13681960. Iola: Krause Publications.

Cuhaj, George S. ( $\left.{ }^{18} 2012\right)$ : Standard Catalog of World Paper Money. Modern Issues. 1961Present. Iola: F \& W Publishing.

Fehlen, Fernand (2013): "Die Stellung des Französischen in Luxemburg. Von der Prestigesprache zur Verkehrssprache". In: Sieburg, Heinz (ed.) (2013): Vielfalt der Sprachen Varianz der Perspektiven. Zur Geschichte und Gegenwart der Luxemburger Mehrsprachigkeit. Bielefeld, Transcript: 37-79.

Gardt, Andreas (2000): "Sprachnationalismus zwischen 1850 und 1945". In: Gardt, Andreas (ed.) (2000): Nation und Sprache. Die Diskussion ihres Verhältnisses in Geschichte und Gegenwart. Berlin/New York, de Gruyter: 247-271.

Grabowski, Hans-Ludwig/Huschka, Henning/Schamberg, Wolfgang (2006): Ausländische Geldscheine unter deutscher Besatzung im Ersten und Zweiten Weltkrieg. Regenstauf: Gietl. 
Hagström, Björn (1984): "Language contact in the Faroes". In: Ureland, Per Sture/Clarkson, Iain (eds.) (1984): Scandinavian language contacts. Cambridge etc., Cambridge University Press: $171-190$.

Hagström, Björn (1992): "Språkkontakt och tvåspråkighet på Färöerna: en översikt". In: Louis-Jensen, Jonna/Poulsen, Jóhan Hendrik W. (eds.) (1992): The Nordic languages and modern linguistics 7/1: 39-60.

Halen, Pierre (2003): "La Belgique: Carrefour des cultures? Essai d'un bilan linguistique et identitaire". In: Schmeling, Manfred (ed.) (2003): Sprache und Identität in frankophonen Kulturen. Opladen, Leske + Budrich: 65-81.

Hansen-Pauly, Marie-Anne (2003): "Der Sprachgebrauch im Alltag der Luxemburger". In: Schmeling, Manfred (ed.) (2003): Sprache und Identität in frankophonen Kulturen. Opladen, Leske + Budrich: 83-100.

Hoff, Tine Adele (2012): Danmark og Farøerne. En historisk undersøgelse af udviklingen $i$ relation mellem Danmark og Farøerne 1850-2010. København: Museum Tusculanum.

Isaksen, Jógvan (1993): Faerøsk litteratur. Introduktion og punktnedslag. Copenhagen Valby: Vindrose.

Jahr, Ernst Håkon (2007): "The planning of modern Norwegian as a sociolinguistic experiment - 'from below'". In: Elspaß, Stephan/Langer, Nils/Scharloth, Joachim/Vandenbussche, Wim (eds.) (2007): Germanic language histories 'from below'. Berlin/New York, de Gruyter: 379-403.

Jahr, Ernst Håkon/Mæhlum, Brit (eds.) (2009): Standardtalemål? Oslo: Novus. (= Themenheft der Norsk Lingvistisk Tidsskrift 27/1)

Janson, Tore (2012): The History of Languages. Oxford: Oxford University Press.

Kramer, Johannes (1996): "Zweieinhalbsprachigkeit (Fallstudien zu Korsika, Curaçao, Seychellen, Gröden, Luxemburg)". In: Marti, Roland (ed.) (1996): Sprachenpolitik in Grenzregionen. Saarbrücken. Saarbrücker Druckerei und Verlag GmbH: 117-135.

Krier, Emile (1976): "Die Außenpolitik des Dritten Reiches gegenüber Luxemburg". In: Funke, Manfred (ed.) (1976): Hitler, Deutschland und die Mächte. Materialien zur Außenpolitik des 3. Reiches. Düsseldorf, Droste: 628-638.

Madsen, Peder S./Christensen, Mogens (1983): Norske pengesedler 1877-1983. Oslo: Selbstverlag.

Mathiak, Robert (1993): "Die rechtliche Stellung der Minderheiten in Belgien". In: Frowein, Jochen Abraham/Hofmann, Rainer/Oeter, Stefan (eds.) (1993): Das Minderheitenrecht europäischer Staaten. Teil 1. Berlin etc., Springer: 1-61.

Nelde, Peter H./Labrie, Normand/William, Colin H. (1992): "The principles of territoriality and personality in the solution of linguistic conflicts". Journal of Multilingual and Multicultural Development 13/5: 387-406.

Nesse, Agnete (2013): Innføring i norsk språkhistorie. Oslo: Cappelen Damm.

Papayian, Eric (2012): "Norge - riket uten rikstalemål?". Norsk Lingvistisk Tidsskrift 30/1: 50-115.

Poulsen, Johan Hendrik W. (1997): "Det færøske sprog". In: Karker, Allan/Lindgren, Birgitta/Løland, Ståle (eds.) (1997): Nordens språk. Oslo, Novus: 177-192.

Schreiner, Patrick (2006): Staat und Sprache in Europa. Nationalstaatliche Einsprachigkeit und die Mehrsprachenpolitik der Europäischen Union. Frankfurt am Main etc.: Lang. 
Steinke, Klaus (2006): "Rumänien und Moldau / Romania and Moldavia". In: Ammon, Ulrich/Dittmar, Norbert/Mattheier, Klaus J./Trudgill, Peter (eds.) (2006): Sociolinguistics / Soziolinguistik: An International Handbook of the Science of Language and Society. 2. Aufl., 3. Teilband. Berlin/New York, de Gruyter: 1818-1823.

Thráinsson, Höskuldur/Petersen, Hjalmar P./Jacobsen, Jógvan í Lon/Hansen, Zakaris Svabo (2004): Faroese. An Overview and Reference Grammar. Tórshavn: Føroya Fróðskaparfelag.

Thomason, Sarah G. (2001): Language Contact. An Introduction. Washington: Georgetown University Press.

Vikør, Lars (2006): "Scandinavia / Skandinavien". In: Ammon, Ulrich/Dittmar, Norbert/Mattheier, Klaus J./Trudgill, Peter (eds.) (2006): Sociolinguistics / Soziolinguistik: An International Handbook of the Science of Language and Society. 2. Aufl., 3. Teilband. Berlin/New York, de Gruyter: 1747-1754.

Vikør, Lars ( $\left.{ }^{3} 2007\right):$ Språkplanlegging. Prinsipp og praksis. Oslo: Novus.

Willemyns, Roland (2006): "The Low Countries / Niederlande". In: Ammon, Ulrich/Dittmar, Norbert/Mattheier, Klaus J./Trudgill, Peter (eds.) (2006): Sociolinguistics / Soziolinguistik: An International Handbook of the Science of Language and Society. 2. Aufl., 3. Teilband. Berlin/New York, de Gruyter: 1758-1765.

Witt, Jörg (2001): Wohin steuern die Sprachen Europas? Probleme der EU-Sprachpolitik. Tübingen: Stauffenburg-Verlag.

Weiller, Raymond (1981): Cent vingt-cinq ans de papier-monnaie luxembourgeois. Luxembourg: BIL.

West, John F. (1972): Faroe. The emergence of a nation. London: Hurst.

Wright, Sue (2012): "Language policy, the nation and nationalism". In: Spolsky, Bernard (ed.) (2012): The Cambridge Handbook of Language Policy. Cambridge, Cambridge University Press: 59-78.

\section{Gesetzestexte}

Heimastýrislógin (1948). http://www.logir.fo/foldb/kunfo/1948/0000011.htm (färöisch) bzw. https://www.retsinformation.dk/Forms/R0710.aspx?id=45897 (dänisch) [23.11.2015].

Forslag til lov om pengesedler i Grønland (2006). http://www.ft.dk/samling/20061/lovforslag/142/index.htm [23.11.2015] .

Lov om Grønlands Selvstyre (2009). http://www.stm.dk/multimedia/selvstyreloven.pdf [23.11.2015].

Lov nr. 248 frá 12. april 1949 om pengesedler m. v. på færøerne (1949). http://www.logir.fo/foldb/lov/1949/0000248.htm [23.11.2015].

Loi du 24 février 1984 sur le régime des langues (1984). http://www.legilux.public.lu/leg/a/archives/1984/0016/a016.pdf [23.11.2015]

Die Verfassung Belgiens (1994). http://www.senate.be/deutsch/const_de.html [23.11.2015]. 